\title{
Nucleon Structure Study at Jefferson Lab
}

\author{
J. P. Chen ${ }^{a}$
}

a Jefferson Lab, Newport News, VA 23606, USA

With high luminosity $6 \mathrm{GeV}$ electron beam, Jefferson Lab (CEBAF)'s study of the nucleon structure is focussed on the regime of strong interaction QCD. Highlights are shown on the study of the nucleon form factors, the spin structure, and the nucleon resonances. Selected new results are presented. Future upgrade to $12 \mathrm{GeV}$ is briefly discussed.

\section{Introduction}

Thomas Jefferson National Accelerator Facility (Jefferson Lab, formally known as CEBAF) has been in operation for over three years. The electron beam has reached the design goal of high intensity (with current up to $200 \mu \mathrm{A}$ ), very low emittance $\left(2 \times 10^{-9}\right)$, and small energy spread $\left(10^{-4}\right)$. The beam energy has increased from it's initial design goal of $4 \mathrm{GeV}$ to $5.5 \mathrm{GeV}$, and is expected to reach $6 \mathrm{GeV}$ in short term. Three simultaneous beams with independent energies and intensities go into three experimental halls, so that three experiments are usually running at same time. High polarization $(\sim 75 \%)$ and high intensity polarized electron beam, polarized targets, and recoil polarimeters make it possible to study physics with spin degree of freedom and to precisely study the nucleon structure by using interference effects. The three experimental halls have complementary detection system. Hall $\mathrm{A}$ and $\mathrm{C}$ have high resolution, high precision spectrometers with associated electron and hadron detectors, suitable for inclusive and semi-inclusive experiments. Hall B has a large acceptance spectrometer, suitable for exclusive and semi-exclusive experiments.

Jefferson Lab is built to study strong interaction in nucleon and nuclei, to understand the transition region between the quark-gluon picture to the nucleon-meson picture. At short distances and short time scales (high energies), coupling involving the elementary quarks and gluons field is weak, and QCD can be solved perturbatively. At large distance, nucleons and mesons are the relevant degrees of freedom. At intermediate distances, quarks and gluons are still relevant but appear as constituent quarks and glue due to confinement. The study at JLab is focussed on this region.

QCD has not been solved for processes at intermediate distance scales and, therefore, the internal structure of nucleons is generally poorly known in this regime. On the other hand, theorists are not challenged due to the lack of high quality data in many areas. The following are areas where the lack of high quality data is most noticeable:

- The electric form factors of the nucleon are poorly known, especially for the neutron. 
- What role do strange quarks play in the nucleon structure?

- The nucleon spin structure has been explored for more than two decades at high energies. The transition from the deep inelastic regime to the confinement regime has not been explored at all. The connection between the GDH and the Bjorken sum rules are to be explored.

- To understand the ground state nucleon we need to understand the full excitation spectrum as well as the continuum. Few transitions to excited states have been studied well, and many states are missing from the spectrum as predicted by our most accepted models.

- The role of the glue in the baryon excitation spectrum is completely unknown, although gluonic excitations of the nucleon are likely produced copiously [1].

Carrying out an experimental program that will address these questions has become feasible due to the availability of high luminosity CW electron accelerator, modern detector instrumentation with high speed data transfer techniques, and the routine availability of spin polarization. Equipped with the above features, Jefferson Lab has become a major contributor to the study of the nucleon structure.

\section{ELASTIC SCATTERING}

\subsection{Electromagnetic form factors}

This process probes the charge and current distribution in the nucleon in terms of the electric $\left(G_{E}\right)$ and magnetic $\left(G_{M}\right)$ form factors. Early experiments in the 1970s on the proton form factor from Bonn, DESY, and CEA showed a violation of the so-called "scaling law", which may be interpreted that the spatial distribution of charge and magnetization are not the same, and the corresponding form factors have different $Q^{2}$ dependencies. The data showed a downward trend for the ratio $R_{E M}=G_{E} / G_{M}$ as a function of $Q^{2}$. Adding the 1989 and 1994 SLAC data sets confuses the picture greatly (Figure 1). Part of the data are incompatible with the other data sets. They also do not show the same general trend as the other data sets. All these data were obtained using the Rosenbluth separation technique and usually have large systematic uncertainties for $G_{E}$, especially at large $Q^{2}$. More precise and reliable data were urgently needed to clarify the experimental situation and to constrain theoretical developments.

The best way to get reliable data at high $Q^{2}$ is via double polarization experiments, and the first experiment[2] of this type has now produced results. Since the ratio $R_{E M}$ is accessed directly in the double polarization asymmetry

$$
A_{\vec{e} \vec{p}}=\frac{k_{1} R_{E M}}{k_{2}\left(R_{E M}\right)^{2}+k_{3}},
$$

this experiment has much lower systematic uncertainties than previous experiments at high $Q^{2}$ (Figure 1). They confirm the trend of the early data, improve the accuracy at high $Q^{2}$ significantly, and extend the $Q^{2}$ range. The data illustrate the power of utilizing polarization in electromagnetic interactions. 
JLab E94-010

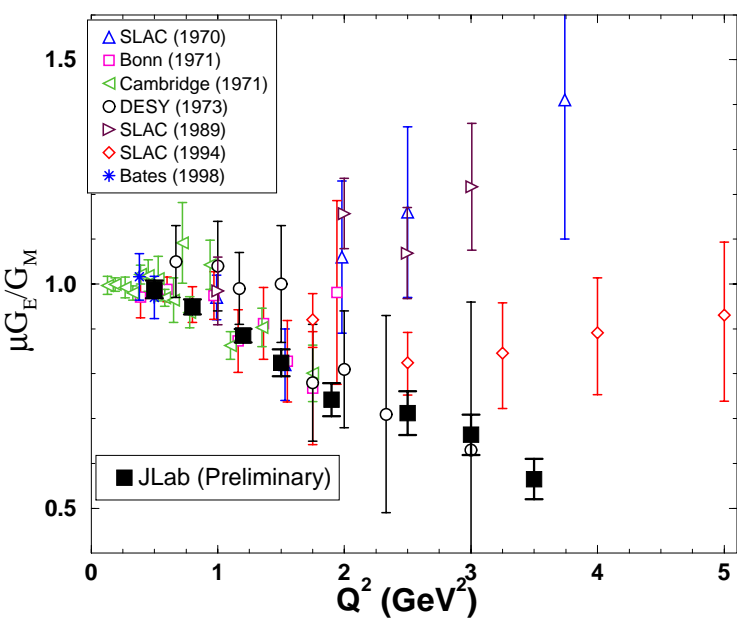

Figure 1. Results for the ratio $R_{E M}^{p}$ of electric and magnetic form factors of the proton. The full squares are preliminary results from JLAB obtained with the double polarization techniques [2]
Figure 2. Preliminary results of raw parallel and perpendicular asymmetries measured in inclusive $\vec{e}^{3} \vec{H} e \rightarrow e X$ scattering at JLAB.

Due to lacking of a free neutron target, the knowledge on the electromagnetic form factors of the neutron is poorer than that of the protons. The knowledge on the neutron magnetic form factor is mostly from using a deuteron target. Polarized ${ }^{3} \mathrm{He}$ target, being approximately a polarized neutron target, provides an alternative way to measure the neutron form factor. A recently completed JLab experiment[3] measured $G_{M}^{n}$ precisely using polarized electron beam on a polarized ${ }^{3} \mathrm{He}$ target.

Few measurements exist on the neutron electric form factor. Several experiments are planned to measure the $G_{E}^{n}$ at JLab with double polarization methods $[4,5]$. Expected results should greatly improve our knowledge of the neutron electrical form factor.

\subsection{Strangeness form factors}

From the analysis of deep inelastic polarized structure function experiments we know that the strange quark sea is polarized, and contributes at the level of a few to $10 \%$ to the nucleon spin. Then one may ask what are the strange quark contributions to the nucleon wave function and their corresponding form factors? The flavor-neutral photon coupling does not distinguish s-quarks from u- or d-quarks. However, the tiny contribution of the $Z^{o}$ is parity violating, and allows measurement of the strangeness contribution. The effect 
is measurable due to the interference with the single photon graph. The asymmetry

$$
A_{\vec{e} p}=\frac{G_{F} Q^{2}}{\sqrt{2} \pi \alpha} \frac{\epsilon G_{E}^{\gamma} G_{E}^{Z}+\tau G_{M}^{\gamma} G_{M}^{Z}}{\epsilon\left(G_{E}^{\gamma}\right)^{2}+\tau\left(G_{M}^{\gamma}\right)^{2}}
$$

in polarized electron scattering contains combinations of electromagnetic and weak form factors which can be expressed in terms of the electromagnetic and the strangeness form factors $\left(G^{s}\right)$. For example, the weak electric form factor can be written:

$$
G_{E}^{Z}=\left(\frac{1}{4}-\sin ^{2} \theta_{W}\right) G_{E p}^{\gamma}-\frac{1}{4}\left(G_{E n}^{\gamma}+G_{E}^{s}\right)
$$

A similar relation holds for the magnetic form factors. The $G^{s}$ form factors can be measured since the $G^{\gamma}$ form factors are known. The elastic $\overrightarrow{e p}$ results of the JLAB HAPPEX experiment [6] measured at $Q^{2}=0.47 \mathrm{GeV}^{2}$ show that strangeness contributions are small, consistent with zero, when measured in a combination of $G_{E}^{s}$ and $G_{M}^{s}$ :

$$
G_{E}^{s}+0.4 G_{M}^{s}=0.023 \pm 0.034(\text { stat }) \pm 0.022(\text { syst }) \pm 0.026\left(G_{E}^{n}\right)
$$

At least a factor of 2 smaller statistical error has been obtained in the new 1999 run, so that the systematic error is limited by our knowledge of the neutron electric form factor! New measurements of $G_{E}^{n}$ should remedy this situation [4,5].

A new precision measurement at $Q^{2}=0.1 \mathrm{GeV}^{2}$ has recently been approved[7] and the expected result will allow us to constrain the strangeness radius to about $5 \%$ level.

Furthermore, a program is planned with a dedicated spectrometer to measure and separate $G_{E}^{s}$ and $G_{M}^{s}$ from $Q^{2}=0.1-1 \mathrm{GeV}^{2}$ with high precision [8].

\section{NUCLEON SPIN STRUCTURE AND GENERALIZED GDH SUM RULE}

The nucleon spin has been of central interest ever since the EMC experiment found that at small distances the quarks carry only a fraction of the nucleon spin. Going from shorter to larger distances the quarks are dressed with gluons and $q \bar{q}$ pairs and acquire more and more of the nucleon spin. How is this process evolving with the distance scale? At the two extreme kinematic regions we have two fundamental sum rules: the Bjorken sum rule (Bj-SR) which holds for the proton-neutron difference in the asymptotic limit, and the Gerasimov Drell-Hearn sum rule (GDH-SR) at $Q^{2}=0$ :

$$
\int_{t h r}^{\infty} \frac{\sigma_{3 / 2}-\sigma_{1 / 2}}{\nu} d \nu=\frac{2 \pi^{2} \alpha}{M^{2}} \kappa^{2}
$$

where $\kappa$ is the anomalous magnetic moment of the nucleon.

Phenomenological models have been proposed to extend the GDH-SR integral for the proton and neutron to finite $Q^{2}$ and connect it to the Bj-SR in deep inelastic regime $[9,10]$. Recently Ji and Osborne[11] has made a rigorous extension of the GDH-SR to the entire region of $Q^{2}$. With the same assumptions as the GDH-SR, Ji and Osborne derived the generalized GDH-SR:

$$
4 \int_{e l}^{\infty} \frac{G_{1}\left(\nu, Q^{2}\right)}{\nu} d \nu=S_{1}\left(Q^{2}\right)
$$


where $S_{1}\left(Q^{2}\right)$ is the forward virtual Compton Scattering amplitude. The GDH-SR and Bj-SR are the two limiting cases $\left(Q^{2}=0\right.$ and $\left.Q^{2}=\infty\right)$ of the generalized GDH-SR. Other than the two limiting cases, $S_{1}\left(Q^{2}\right)$ can also be calculated at small $Q^{2}$, where hadrons are the relevant degree of freedom, with Chiral Perturbation theory and at large $Q^{2}$, where quarks and gluons(partons) are the relevant degree of freedom, with higher order QCD expansion (twist expansion). At small $Q^{2}$, it was calculated to the leading order[11] using the Chiral Perturbation theory with the Heavy Baryon approximation. At large $Q^{2}$, twist-2 and twist-4 terms have been calculated. Efforts are underway to calculate to higher orders for both small and large $Q^{2}$.

A crucial question in this connection is how low in $Q^{2}$ the Bj-SR can be evolved using the high twist expansion? Recent estimates [11] suggest as low as $Q^{2}=0.5 \mathrm{GeV}^{2}$. Also at the other end, chiral perturbation theory is applicable at small $Q^{2}$, and may allow evolution of the GDH-SR to $Q^{2}=0.02 \mathrm{GeV}^{2}$ or higher. Theoretical efforts (such as lattice calculations) are needed to bridge the remaining gap.

The importance of such efforts cannot be overemphasized as it would mark the first time that hadronic structure is described by a fundamental theory in the entire kinematics regime, from short to large distances.

Experiments have been carried out at JLAB on polarized $N H_{3}[12], N D_{3}[13]$, and ${ }^{3} \mathrm{He}[14]$ targets to extract the $Q^{2}$ evolution of the GDH integral for protons and neutrons in a range of $Q^{2}=0.1-2.0 \mathrm{GeV}^{2}$ and from the elastic to the deep inelastic regime. Results on the Sum Rule are expected in 2000. Figure 2 shows some preliminary raw asymmetry results from the experiment on polarized ${ }^{3} \mathrm{He}$. Both parallel (target polarized along beam direction) and perpendicular (target polarized perpendicular to the beam direction) asymmetries are shown. The parallel asymmetries are positive in the quasielastic peak, negative in the $\Delta$ region, and become small in the higher mass resonances. Contrast to high energy case, the perpendicular asymmetries are as large in amplitude as the parallel asymmetry at this low energy.

A new experiment[15] will extend the range down to very low $Q^{2}$ (below $0.02 \mathrm{GeV}^{2}$ ) and to higher virtual photon energies in order to extrapolate to the real GDH-SR.

\section{EXCITATION OF NUCLEON RESONANCES}

A large effort is being extended to the study of excited states of the nucleon. The transition form factors contain information on the spin structure of the transition and the wave function of the excited state. We test predictions of baryon structure models and strong interaction QCD. Another aspect is the search for, so far, unobserved states which are missing from the spectrum but are predicted by the QCD inspired quark model [16]. Also, are there other than $\mid Q^{3}>$ states? Gluonic excitations of the nucleon, i.e. $\mid Q^{3} G>$ states should be copious, and some resonances may be "molecules" of baryons and mesons $\mid Q^{3} Q \bar{Q}>$. Finding at least some of these states is important to clarify the intrinsic quark-gluon structure of baryons and the role played by the glue and mesons in hadron spectroscopy and structure. Electroproduction is an important tool in these studies as it probes the internal structure of hadronic systems.

The scope of the $N^{*}$ program[17] at JLAB is to measure many of the possible decay channels of resonances in a large kinematics range. 
Most of the JLab $N^{*}$ program are in Hall B using the the large acceptance (CLAS) detector. The goal is to provide data in the entire resonance region, by measuring most channels, with large statistics, including many polarization observables. First round of experiments are being carried out and data analyses are underway. The data quality of some initial preliminary results already show often order of magnitude improvement over the existing data. Some interesting features started to show up. Exciting results are expected in the next few years.

\section{QUTLOOK}

The ongoing experimental effort will provide us with a wealth of data in the next decade to address many open problems in hadronic structure at intermediate distances. The experimental effort must be accompanied by a significant theoretical effort to translate this into real progress in our understanding of the complex regime of strong interaction physics.

To accommodate new physics requirements, an energy upgrade in the 10-12 GeV range has been proposed for the CEBAF machine at JLab. This upgrade will be accompanied by the construction of a new experimental hall for tagged photon experiments with a $4 \pi$ solenoid detector to study exotic meson spectroscopy, and production of other heavy mesons. Existing spectrometers and detectors in the other three Halls will be upgraded to match the upgraded energy accelerator. Other than the hadron specotroscopy study in search of the gluonic degree of freedom and other exotic mesons and baryons, the energy upgrade also provides a much greater kinematic regime for the deep inelastic scattering and higher $Q^{2}$ range for the elastic and the resonance region. We will be in an ever better position to study the nucleon structure and strong interaction in the intermediate distance.

\section{REFERENCES}

1. N. Isgur, hep-ph/9904494, (1999)

2. M. Jones, et al., submited to Phys. Rev. Lett., 1999

3. JLab E95-001, Spokespersons, H. Gao and O. Hansen

4. JLab E93-026, Spokespersons, D. Day and J. Mitchell

5. JLab E93-038, Spokespersons, R. Madey and S. Kowalski

6. K. A. Aniol, et al., Phys. Rev. Lett. 82, 1096 (1999)

7. JLab E99-115, Spokespersons, K. Kumar and D. Lhuillier

8. JLab E99-016, Spokesperson, D. Beck

9. M. Anselmino, B. Ioffe and E. Leader, Sov. J. Nucl. Phys. 49, 136 (1989)

10. V. Burkert and B. Ioffe, JETP, Vol. 105, 1153 (1994)

11. X. Ji and J. Osborne, hep-ph/9905010 (1999)

12. JLab E91-023, Spokespersons, V. Burkert, D. Crabb and R. Minehart

13. JLab E93-009, Spokespersons, S. Kuhn and M. Taiuti

14. JLab E94-010, Spokespersons, Z. E. Meziani, G. Cates and J. P. Chen

15. JLab E97-110, Spokespersons, J. P. Chen, G. Cates and F. Garibaldi

16. N. Isgur, G. Karl, Phys. Rev. D23, 817 (1981)

17. V.D. Burkert, Nucl. Phys. A623 (1997) 59c-70c 